\title{
Is Solar Wind electron precipitation a source of neutral heating in the nightside Martian upper atmosphere?
}

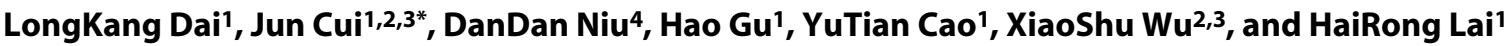 \\ 'Planetary Environmental and Astrobiological Research Laboratory (PEARL), School of Atmospheric Sciences, Sun Yat-sen University, Zhuhai Guangdong \\ 519082, China; \\ ${ }^{2}$ Key Laboratory of Lunar and Deep Space Exploration, National Astronomical Observatories, Chinese Academy of Sciences, Beijing 100101, China; \\ ${ }^{3}$ Center for Excellence in Comparative Planetology, Chinese Academy of Sciences, Hefei 230031, China; \\ ${ }^{4}$ Institute of Space Science and Applied Technology, Harbin Institute of Technology, Shenzhen Guangdong 518055, China
}

\section{Key Points:}

- We derive the temperature profiles in the nightside Martian upper atmosphere.

- Evidence for solar wind heating is rare in the nightside Martian upper atmosphere.

- Evidence for nightside solar wind heating is more prominent below $160 \mathrm{~km}$.

Citation: Dai, L. K., Cui, J., Niu, D. D., Gu, H., Cao, Y. T., Wu, X. S. and Lai, H. R. (2021). Is Solar Wind electron precipitation a source of neutral heating in the nightside Martian upper atmosphere?. Earth Planet. Phys., 5(1), 1-10. http://doi.org/10.26464/epp2021012

\begin{abstract}
Solar Wind (SW) electron precipitation is able to deposit a substantial amount of energy in the nightside Martian upper atmosphere, potentially exerting an influence on its thermal structure. This study serves as the first investigation of such an issue, with the aid of the simultaneous measurements of both neutral density and energetic electron intensity made on board the recent Mars Atmosphere and Volatile Evolution (MAVEN) spacecraft. We report that, from a statistical point of view, the existing measurements do not support a scenario of noticeable neutral heating via SW electron precipitation. However, during $3 \%-4 \%$ of the MAVEN orbits for which data are available, strong correlation between nightside temperature and electron intensity is observed, manifested as collocated enhancements in both parameters, as compared to the surrounding regions. In addition, our analysis also indicates that neutral heating via SW electron precipitation tends to be more effective at altitudes below $160 \mathrm{~km}$ for integrated electron intensities above $0.01 \mathrm{ergs} \cdot \mathrm{cm}^{-2} \cdot \mathrm{s}^{-1}$ over the energy range of 3-450 eV. The results reported here highlight the necessity of incorporating SW electron precipitation as a heat source in the nightside Martian upper atmosphere under extreme circumstances such as during interplanetary coronal mass ejections.
\end{abstract}

Keywords: Mars; Solar Wind; upper atmosphere; MAVEN

\section{Introduction}

The thermal structure of the dayside Martian upper atmosphere is well known to be driven by solar Extreme Ultraviolet (EUV) heating (e.g. Forbes et al., 2008; Krasnopolsky, 2010), a scenario that is supported by recent measurements made with several instruments on board the Mars Atmosphere and Volatile Evolution (MAVEN) spacecraft (Jakosky et al., 2015). Mean neutral temperatures are readily obtained from the observed scale heights of the total atmospheric density measured by the Accelerometer (Zurek et al., 2017), the densities of various atmospheric constituents measured by the Neutral Gas and lon Mass Spectrometer (NGIMS) (Mahaffy et al., 2015a; Stone et al., 2018; Cui J et al., 2018), as well as the intensities of distinctive airglow emission features, such as the $\mathrm{CO}_{2}^{+}$ultraviolet double emission and the $\mathrm{CO}$ Cameron band

Correspondence to: J. Cui, cuijun7@mail.sysu.edu.cn

Received 14 SEP 2020; Accepted 26 NOV 2020.

Accepted article online 25 DEC 2020.

(C)2021 by Earth and Planetary Physics. emission, measured by the Imaging Ultraviolet Spectrograph (Jain et al., 2015, 2018). The combined analysis of Bougher et al. (2017) confirmed that the temperatures estimated from different data sources are in agreement and reveal pronounced variations with solar EUV irradiance and heliocentric distance. In addition, existing evidence also suggests that the role of solar forcing may sometimes be overwhelmed by other forcing processes, such as atmospheric waves and tides (e.g. Forget et al., 2009; Stiepen et al., 2015).

In the nightside Martian upper atmosphere where the solar radiation is switched off, energetic electron precipitation, primarily of Solar Wind (SW) origin, has been considered as the dominant source of energy deposition. For instance, it has been proposed that SW electron precipitation is responsible for the formation of a patchy and sporadic nightside ionosphere on Mars (e.g. Cui J et al., 2015, 2019; Girazian et al., 2017), that it causes auroral emission (Bertaux et al., 2005), and that it possibly powers nightside atmospheric escape as well (e.g. Zhang Q et al., 2020). SW electron precipitation may also exert an influence on the thermal structure 
of the nightside Martian upper atmosphere. Historically, the thermal structure has not been well constrained by observations on the nightside of Mars (Schofield et al., 1997; Magalhães et al., 1999) and the role of energetic electron precipitation has consequently not been explored in detail. Indeed, no thermospheric global circulation model calculations to date have incorporated electron precipitation as a potential external energy source (e.g. Bougher and Robel, 1991; Bougher et al., 1999, 2000; Angelats i Coll et al., 2005; González-Galindo et al., 2005, 2009a, b).

Fortunately, our knowledge of the thermal structure of the nightside Martian upper atmosphere has been greatly improved over the past few years by the accumulation of MAVEN NGIMS measurements. The recent NGIMS investigation of Stone et al. (2018) revealed a mean nightside temperature of $127 \pm 8 \mathrm{~K}$ in the Martian upper atmosphere, which was about $50 \%$ of the mean subsolar temperature of $260 \pm 7 \mathrm{~K}$. In addition, the analysis of Pilinski et al. (2018), also using the NGIMS data, identified several persistent nightside temperature structures near the dusk and dawn terminators of Mars, which were interpreted as caused either by nightside dynamical heating associated with converging and downwelling winds or by terminator waves originating in the lower atmosphere. Complementary to the NGIMS temperature data, the Solar Wind Electron Analyzer (SWEA) on board MAVEN (Mitchell et al., 2016) is able to make simultaneous measurements of energetic electron intensity in the vicinity of Mars, thus allowing the role of SW electron precipitation on nightside thermal structure to be explored for the first time. This serves as the main motivation of the present study.

\section{Data Description and Extraction of Neutral}

\section{Temperature}

Neutral temperatures in the nightside Martian upper atmosphere can be readily retrieved from the NGIMS measurements either via isothermal fitting to the neutral density data (e.g. Mahaffy et al., 2015a) or via downward integration of the momentum equation of an atmospheric species (e.g. Stone et al., 2018).

The NGIMS is a quadrupole mass analyzer capable of measuring the densities of various constituents of the Martian upper atmosphere and ionosphere over the mass range of 2-150 Da with a mass resolution of 1 Da (Mahaffy et al., 2015b). The neutral temperature analysis presented here relies exclusively on NGIMS measurements in the Closed Source Neutral mode from October 2014 to October 2018. The available NGIMS data typically cover the regions of the Martian upper atmosphere from $250 \mathrm{~km}$ down to $150 \mathrm{~km}$ during the nominal MAVEN mission phase and down to $120 \mathrm{~km}$ during isolated Deep Dip campaigns. In this study, the nightside regions are defined as those with solar zenith angle (SZA) greater than $110^{\circ}$, corresponding to the regions fully within the optical shadow from the MAVEN periapsis up to the mean exobase altitude of $200 \mathrm{~km}$ on the nightside of Mars (Fu MH et al., 2020). For comparison, we define the dayside and near-terminator regions as those with $\mathrm{SZA}<90^{\circ}$ and $90^{\circ}<\mathrm{SZA}<110^{\circ}$, respectively. The entire dataset contains 1700 dayside orbits, 550 nearterminator orbits, and 570 nightside orbits.

For each MAVEN orbit, the available NGIMS density measure- ments allow neutral temperatures to be derived from six individual species: $\mathrm{CO}_{2}, \mathrm{O}, \mathrm{N}_{2}, \mathrm{CO}, \mathrm{Ar}$, and He (Mahaffy et al., 2015a). This requires that the distribution of $\mathrm{CO}_{2}$, as the dominant species, be in hydrostatic equilibrium and the distribution of the remaining species be in diffusive equilibrium. For the above reason, He is not considered because it is a relatively light species possibly subject to strong escape (Bovino et al., 2011; Gu H et al., 2020a). In addition, $\mathrm{CO}$ is also not considered because it has been argued that the NGIMS CO densities are unreliable due to incomplete knowledge of the relevant cracking patterns (Wu XS et al., 2020).

For the remaining four species, $\mathrm{CO}_{2}, \mathrm{O}, \mathrm{N}_{2}$, and $\mathrm{Ar}$, their NGIMS densities are likely to be subject to the well-known effect of wall contamination (e.g. Cui J et al., 2009), as displayed in Figure 1, comparing the mean temperatures derived from isothermal fittings to the inbound and outbound density measurements separately. The figure suggests clearly that the outbound temperatures are substantially higher than the inbound temperatures for $\mathrm{CO}_{2}$ and $\mathrm{O}$, a feature that has been attributed to wall contamination rather than realistic variations of the ambient atmosphere (e.g. Fox et al., 2017; Fu MH et al., 2020). In contrast, the inbound and outbound temperatures from $\mathrm{N}_{2}$ and Ar are nearly identical, indicative of negligible wall contamination. For this study, the NGIMS Ar densities are used to infer the thermal structure of the nightside Martian upper atmosphere, including both the inbound and outbound measurements. Compared with some previous investigations relying on the outbound NGIMS $\mathrm{CO}_{2}$ densities only (e.g. Cui J et al., 2018), using the NGIMS Ar densities effectively doubles the available sample size.

To get an overview of the thermal structure of the nightside Martian upper atmosphere, we show in Figure 2 the nightside temperature distribution based on isothermal fittings to NGIMS $\mathrm{Ar}$ density data from the periapsis altitudes (typically $150 \mathrm{~km}$ ) up to $200 \mathrm{~km}$ during all available MAVEN orbits. The dayside and nearterminator temperature distributions are also shown for comparison where we treat the dawn and dusk terminators separately. According to the NGIMS measurements, the median temperature in the Martian upper atmosphere declines from $200 \mathrm{~K}$ on the dayside to $125 \mathrm{~K}$ on the nightside, whereas the dawn and dusk terminator temperatures have comparable mean values of $157 \mathrm{~K}$ and $163 \mathrm{~K}$, respectively. The scattering in temperature is minimized as $20 \%$ on the dayside, increases slightly to $25 \%$ near the dusk terminator and on the nightside, and becomes maximized as $35 \%$ near the dawn $25 \%$ terminator. The mean nightside temperature that we obtain is fully consistent with the value of $127 \mathrm{~K}$ reported in Stone et al. (2018).

During each MAVEN orbit, two individual neutral temperature profiles can also be derived from the NGIMS Ar data, one from inbound and the other one from outbound, via the downward integration of the diffusive equilibrium equation in the form of

$$
\frac{\mathrm{d}}{\mathrm{d} r}(n k T)=\frac{-G M m n}{r^{2}}
$$

where $n$ is the Ar number density, $k$ is the Boltzmann constant, $T$ is the neutral temperature, $m$ is the Ar atomic mass, $G$ is the gravitational constant, $M$ is the planetary mass, and $r$ is the planetocentric radius. By way of illustration, Figure 3 displays an example 

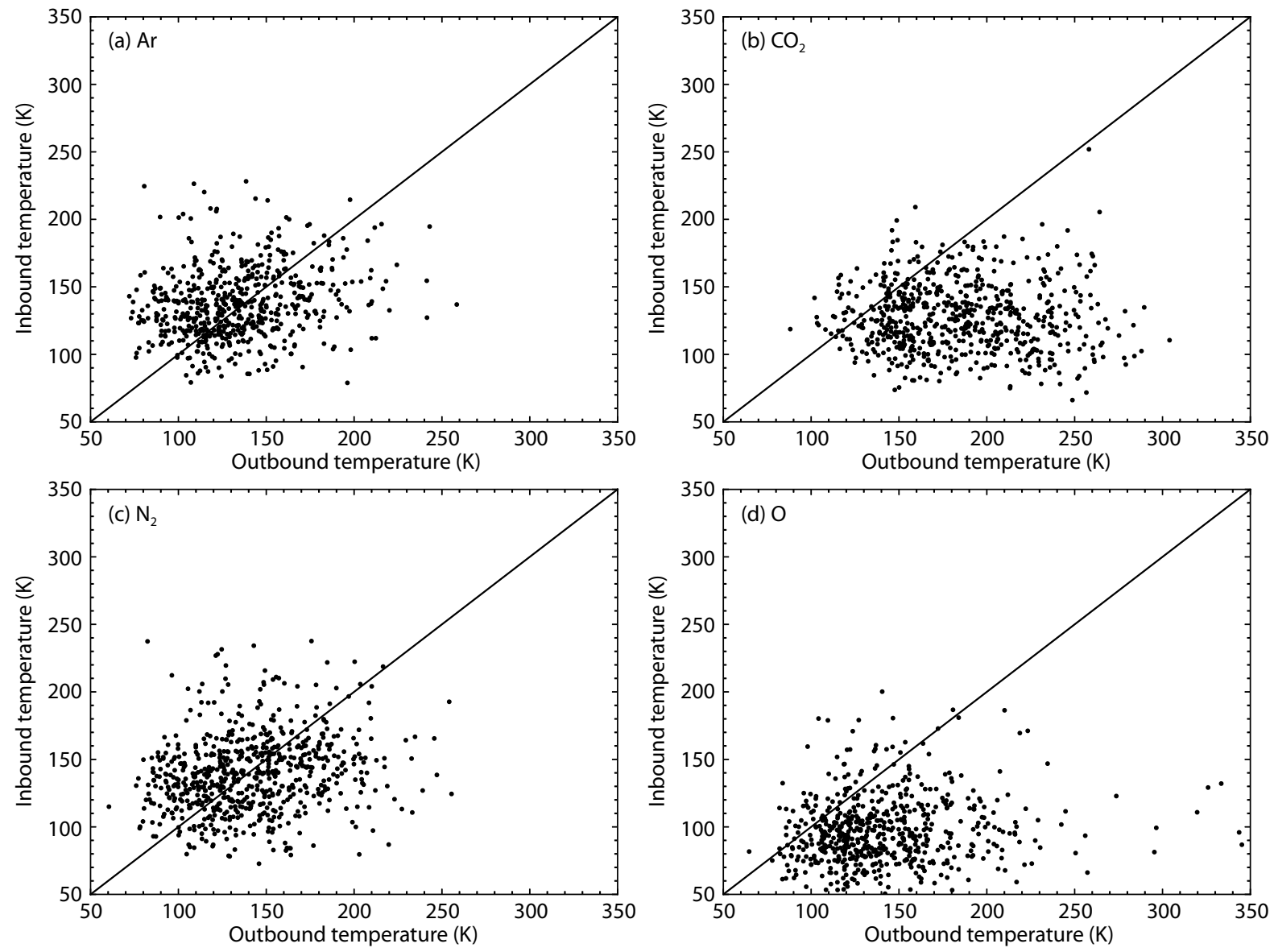

Figure 1. A comparison between the inbound and outbound isothermal temperatures inferred from the MAVEN NGIMS measurements of Ar, $\mathrm{CO}_{2}, \mathrm{~N}_{2}$, and $\mathrm{O}$, respectively.

of the nightside Ar density profile obtained during the inbound portion of MAVEN orbit \#1600, along with the respective temperature profile derived from Equation (1), assuming an upper boundary temperature of $100 \mathrm{~K}$.

Several caveats need to be emphasized:

(1) The derived temperature profile depends somewhat on the choice of the upper boundary temperature at $200 \mathrm{~km}$, but previous investigations have indicated that this arbitrary choice has very little impact on the temperatures at one scale height below the upper boundary (e.g. Cui J et al., 2018).

(2) The effect of eddy mixing has been implicitly ignored in deriving the temperature profile, which is a reasonable approximation because the homopause altitude on Mars is usually located well below the MAVEN periapsis (e.g. Slipski et al., 2018).

(3) Temperature extraction using Equation (1) relies on the assumption that the observed Ar density variation along the spacecraft trajectory is purely vertical, which means that the horizontal variation in the Martian upper atmosphere has been neglected (e.g. Stone et al., 2018).

(4) Recently, Leclercq et al. (2020) have argued that the procedure of downward integration is likely to fail in the presence of wave activity, but this should not affect the temperatures averaged over a large number of orbits, provided that the phase shifts between different waves are random.

In Figure 4, we show the temperature profiles derived from NGIMS Ar density data acquired during the inbound portions of several representative nightside MAVEN orbits. In each panel, the thick solid line corresponds to the case with the upper boundary temperature taken to be the mean temperature at $200-250 \mathrm{~km}$ from isothermal fitting, whereas the thin solid lines show the temperature profiles with the upper boundary temperatures shifted by $-20 \mathrm{~K},-10 \mathrm{~K},+10 \mathrm{~K}$, and $+20 \mathrm{~K}$, respectively. The figure clearly demonstrates that for each case, a convergency is reached among different temperature profiles at $10 \mathrm{~km}$ below the upper boundary. Distinctive wavelike features are clearly seen in the figure, indicative of the omnipresence of gravity waves in the Martian upper atmosphere (e.g. England et al., 2017; Siddle et al., 2019). It is also noteworthy that the temperatures at the lowest altitude obtained from the inbound and outbound data for each orbit are usually not identical despite the fact that they essentially characterize the same atmospheric regions. This must be connected to horizontal variations that have been implicitly neglected in downward integration (e.g. Stone et al., 2018).

Finally, we discuss the uncertainties in temperatures derived both from isothermal fitting and from downward integration, which are crucial for a rigorous identification of the potential impact of energetic electron precipitation on the thermal structure of the nightside Martian upper atmosphere. Typically, the temperature uncer- 

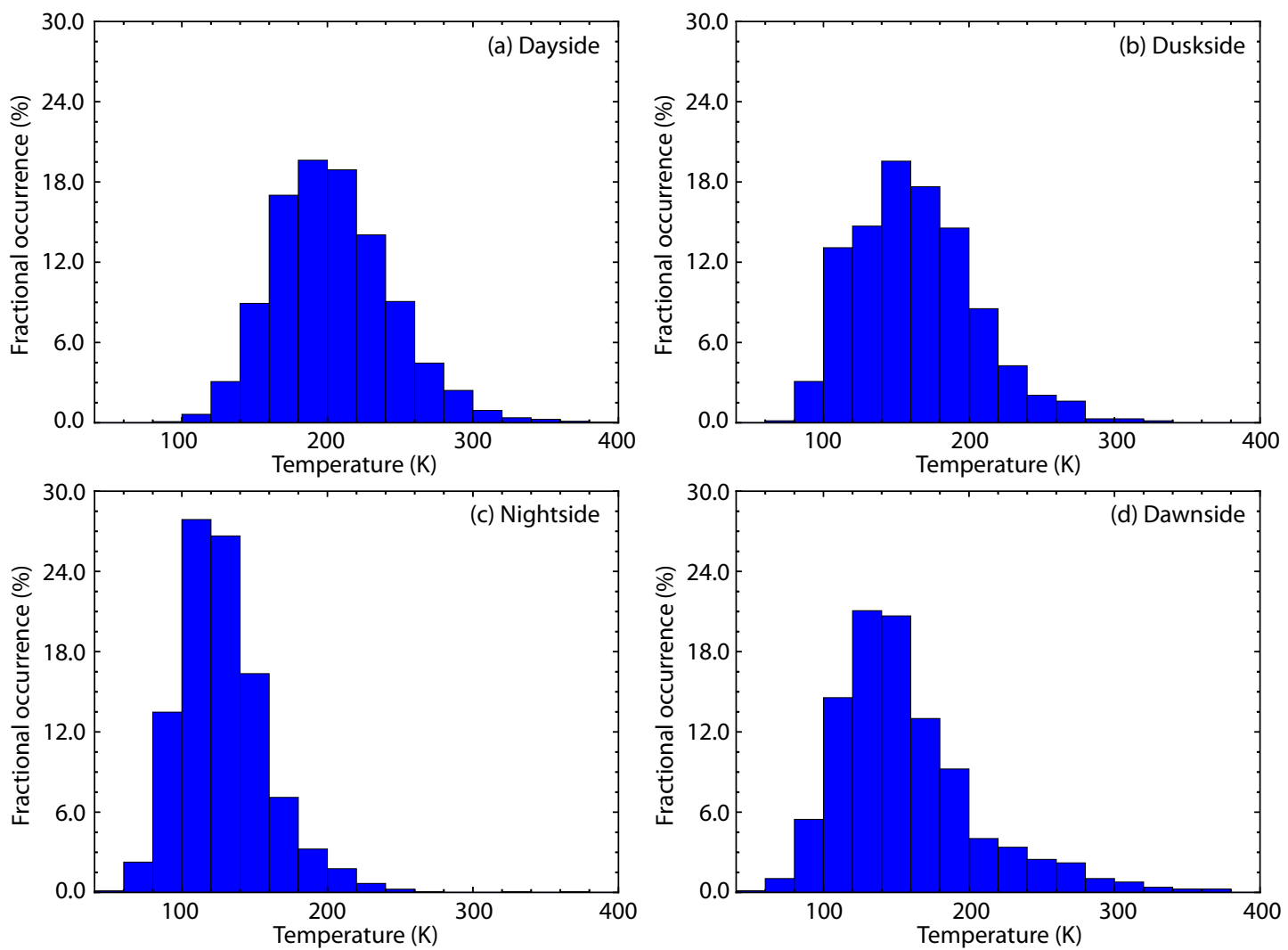

Figure 2. A comparison of the temperature distribution in the Martian upper atmosphere: (a) dayside, (b) duskside, (c) nightside, and (d) dawnside. The temperatures are obtained from isothermal fittings to the NGIMS Ar density data, both inbound and outbound, from the periapsis altitudes up to $200 \mathrm{~km}$ during all available MAVEN orbits.
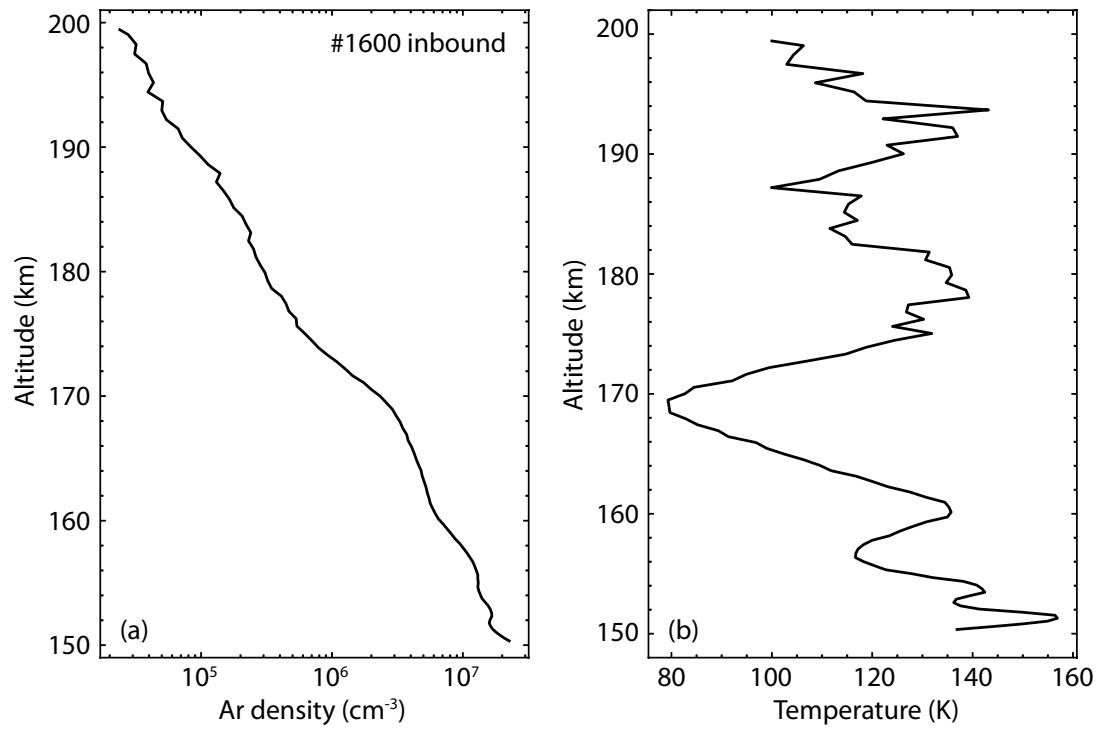

Figure 3. The nightside Ar density profile measured by the NGIMS during the inbound portion of MAVEN orbit \#1600 (a), along with the respective temperature profile derived by downward-integrating the diffusive equilibrium equation assuming an upper boundary temperature of $100 \mathrm{~K}(\mathrm{~b})$.

tainty near and above $190 \mathrm{~km}$ is likely to be large and due mainly to the unknown upper boundary temperature, whereas at relatively low altitudes, the temperature uncertainty is $10 \mathrm{~K}$ and is dominated by horizontal variations in the Ar density (Stone et al., 2018).

\section{Correlation Between Energetic Electron Precipitation and Thermal Structure}

Valuable information on SW energetic electron precipitation in the vicinity of Mars is collected by the MAVEN SWEA, a symmetric 

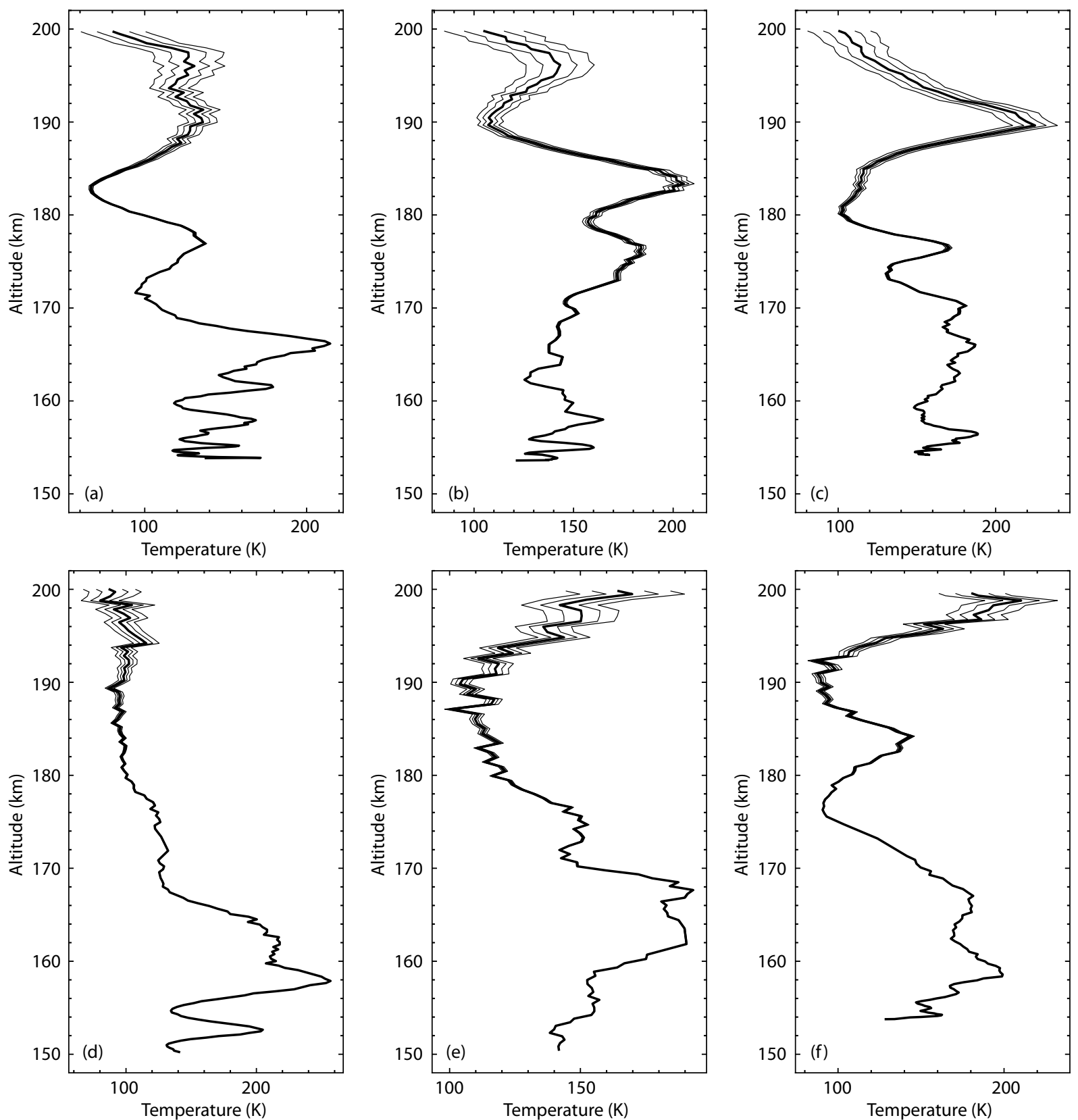

Figure 4. The nightside temperature profiles derived from NGIMS Ar density data acquired during the inbound portions of MAVEN orbits $\# 368$ (a), \#387 (b), \#408 (c), \#1596 (d), \#3371 (e), and \#4512 (f), all obtained by downward-integrating the diffusive equilibrium equation with the effect of eddy mixing ignored. In each panel, the thick solid line corresponds to the case with the upper boundary temperature taken to be the mean temperature at $200-250 \mathrm{~km}$ from isothermal fitting, whereas the thin solid lines show the profiles with the upper boundary temperatures shifted by $-20 \mathrm{~K},-10 \mathrm{~K},+10 \mathrm{~K}$, and $+20 \mathrm{~K}$, respectively.

hemispheric electrostatic analyzer that measures electron intensity over the energy range of $3-4.6 \mathrm{keV}$ with a resolution of $17 \%$ (Mitchell et al., 2016). The instrument is also capable of measuring the arrival direction of energetic electrons within a field of view (FOV) of $360^{\circ} \times 120^{\circ}$ of which $8 \%$ is blocked by the spacecraft body (Mitchell et al., 2016).

Energetic electron precipitation in the nightside Martian upper atmosphere is notably characterized by the appearance of electron depletion that has been shown to be strongly modulated by the ambient magnetic field configuration (e.g. Steckiewicz et al., 2015,
2017; Niu DD et al., 2020). This means that if SW electron precipitation indeed serves as a significant source of neutral heating, a visible correlation between neutral temperature and crustal magnetic field intensity should be revealed by existing observations. Especially, reduced temperatures near the strong crustal magnetic fields that are preferentially located over the southern hemisphere of Mars (Acuña et al., 1998; Connerney et al., 1999) are expected. However, this is not the case as indicated in Figure 5 where we present the geographic distribution of isothermal temperature at 150-200 km superimposed on the crustal magnetic field model of Morschhauser et al. (2014) appropriate for an alti- 
tude of $400 \mathrm{~km}$. We therefore conclude that from a statistical point of view, SW electron precipitation does not exert a significant impact on the thermal structure of the nightside Martian upper atmosphere.

A similar conclusion could also be reached in a more direct manner by inspecting the variation of the nightside neutral temperature as a function of the integrated energetic electron intensity over the same vertical column of 150-200 km, over the energy range of $3-450 \mathrm{eV}$, as well as over the full instrument FOV. The lower energy limit corresponds to the minimum electron energy measured by the SWEA, ignoring the spacecraft charging effect (Mitchell et al., 2016), at which SW electron precipitation could deposit energy locally via impact excitation of atmospheric neutrals (e.g. Gu H et al., 2020b). The upper energy limit corresponds to where the measured electron intensity on the nightside of Mars drops rapidly to the noise level (Niu DD et al., 2020). The variation between the nightside temperature and integrated electron intensity is depicted in Figure 6, essentially revealing no correlation. Note that the uncertainties in both isothermal neutral temperature and integrated electron intensity are typically very small and are thus not shown in the figure.

In Figure 7, we compare further the NGIMS-derived temperature profiles and the SWEA-derived electron energy spectra (in terms of the omnidirectional electron intensity), accumulated during several representative nightside MAVEN orbits as indicated in the figure legend with " $\mathrm{i}$ " standing for inbound and " $\mathrm{O}$ " standing for outbound. The displayed temperature profiles are those obtained with the upper boundary temperatures set as the mean temperatures at 200-250 km, during these orbits (see Section 3). Figure 7 clearly suggests a multifaceted thermal structure in the nightside Martian upper atmosphere, which we describe in detail below.

The top row of Figure 7 presents four examples, orbits \#1605 (i) in panel (a), \#3353 (i) in panel (b), \#3623 (o) in panel (c), and \#4495 (i) in panel (d), for which the NGIMS-based temperature profiles

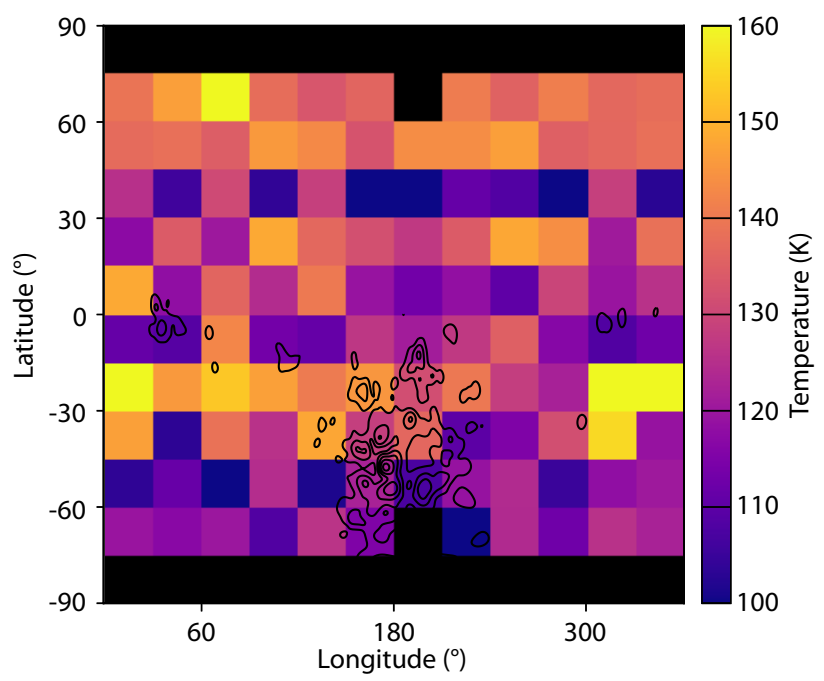

Figure 5. The geographic distribution of isothermal temperature at 150-200 km in the nightside Martian upper atmosphere, superimposed on the crustal magnetic field model of Morschhauser et al. (2014) appropriate for an altitude of $400 \mathrm{~km}$. demonstrate distinctive structures uncorrelated with the SWEAbased energetic electron intensity measurements made during the same orbits. For the first example, strong temperature fluctuations are seen, which are more likely associated with atmospheric gravity waves widely observed in the Martian upper atmosphere (e.g. England et al., 2017; Siddle et al., 2019). For comparison, electron precipitation displays a quite different pattern, most pronounced above $190 \mathrm{~km}$ where the temperature drops to a minimum. For the second example, the nightside temperature increases nearly monotonically from $165 \mathrm{~km}$ towards the lower boundary, to be distinguished from the observed energetic electron precipitation with an isolated enhancement covering $10 \mathrm{~km}$ centered around $160 \mathrm{~km}$. Similar situations are encountered for the remaining two examples, each demonstrating a continuous band of electron precipitation extending to at least $200 \mathrm{~km}$ whereas the temperature structure is distinctly characterized by the presence of several localized temperature enhancements.

In the bottom row of Figure 7, four additional examples are presented including MAVEN orbits \#474 (i) in panel (e), \#3425 (i) in panel (f), \#3794 (o) in panel (g), and \#4464 (i) in panel (h), all with apparent correlations between nightside temperature and energetic electron intensity. For the first example, three localized temperature enhancements are observed near 165, 180, and $200 \mathrm{~km}$, where simultaneous enhancements in electron intensity are also observed. For the second example, the nightside temperature is enhanced over the altitude range of $155-175 \mathrm{~km}$ with two localized maxima near 160 and $170 \mathrm{~km}$, collocated with a continuous band of substantial energetic electron precipitation that also exhibits two localized maxima at similar altitudes. The third example is characterized by a consistent pattern of nightside thermal structure and electron precipitation with three localized enhancements near 165, 170, and $190 \mathrm{~km}$. For the last example, an apparent correlation between nightside temperature and electron intensity is also suggested by the MAVEN data revealing simultaneous enhancements near 180 and $190 \mathrm{~km}$.

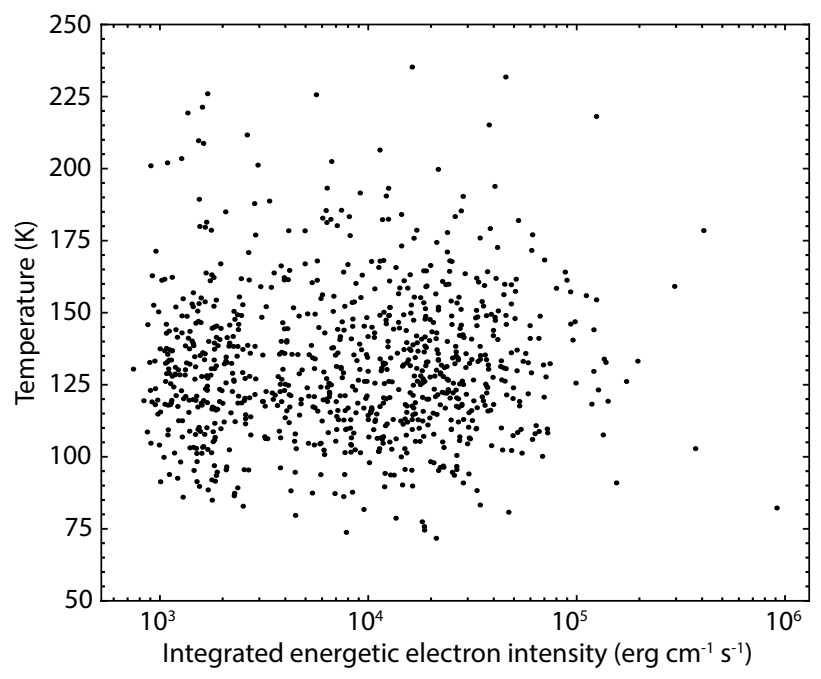

Figure 6. Isothermal neutral temperature in the nightside Martian upper atmosphere as a function of the energetic electron intensity integrated over the altitude range of 150-200 km, the energy range of $3-450 \mathrm{eV}$, and the full instrument FOV. 

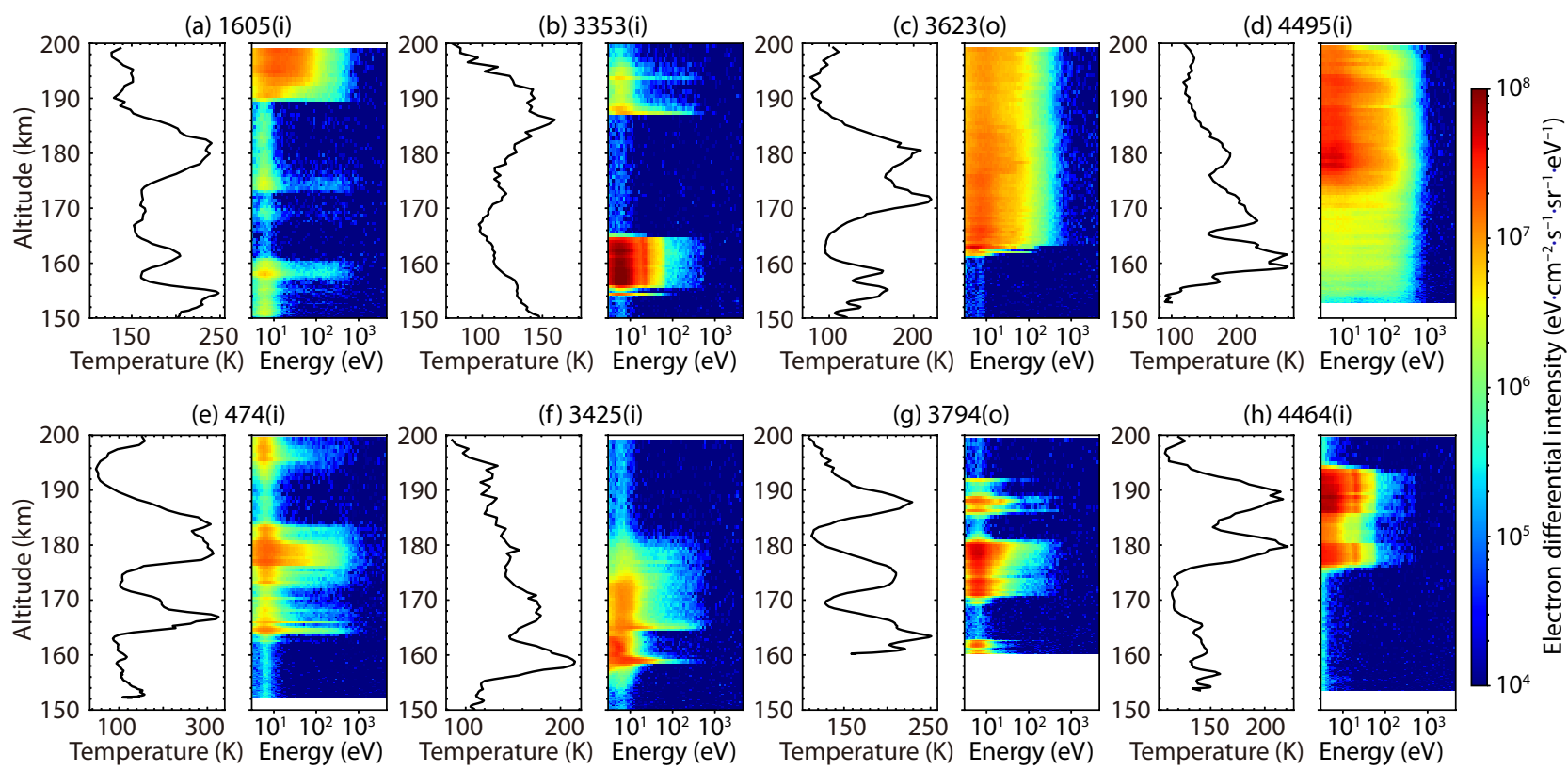

Figure 7. A comparison between the NGIMS-derived temperature profiles and the SWEA-derived electron energy spectra (in terms of the omnidirectional electron intensity), accumulated during several representative nightside MAVEN orbits as indicated in the figure legend with " $i$ " standing for inbound and " $\mathrm{O}$ " standing for outbound, respectively. The displayed temperature profiles are obtained by downward-integrating the Ar diffusive equilibrium equation with upper boundary temperatures set as the mean temperatures at 200-250 km.

By scrutinizing the entire data set, we find that the majority of MAVEN orbits show observations similar to those in the top row of Figure 7 , in which the neutral temperature is uncorrelated with energetic electron intensity, whereas only 3.5\% show apparent correlations between the two parameters. The identification of correlation is based on visual inspection of coexisting localized enhancements in both NGIMS-based temperature and SWEAbased electron intensity, a necessarily imprecise procedure that is clearly subject to some uncertainties. Despite this, the results presented here undoubtedly indicate that observational evidence for the possible impact of SW electron precipitation on nightside temperature is rare. It is also noteworthy that the nightside temperature can rise to $200 \mathrm{~K}$ or higher, which is well above the nightside mean temperature of $125 \mathrm{~K}$, but when it does so, it is associated observationally with enhanced SW electron precipitation. As mentioned at the end of Section 2, the temperature uncertainty from downward integration is $10 \mathrm{~K}$, which is much smaller than the typical temperature enhancement seen in Figure 7.

The analysis presented so far relies on collocated enhancement in both temperature and electron intensity on the nightside of Mars, but this represents only one aspect of the SW impact on the atmospheric thermal structure. For further investigation, we divide all available nightside SWEA measurements made at any given altitude into several consecutive bins with different ranges of electron intensity, and we compute the respective mean nightside temperature for each bin based on simultaneous NGIMS Ar measurements. The corresponding mean nightside temperature profiles for different bins are displayed in Figure 8, revealing a pronounced temperature enhancement below $160 \mathrm{~km}$ for the highest electron intensity bin of $0.01-0.1 \mathrm{ergs} \cdot \mathrm{cm}^{-2} \cdot \mathrm{s}^{-1}$, where the electron intensity is obtained by integrating the SWEA spectrum over the energy range of $3-450 \mathrm{eV}$ (see above) and over the full instrument FOV. This indicates that the impact of SW electron precipitation tends to be effective at relatively low altitudes, only when the ambient electron intensity is sufficiently high. We also caution that the scattering in temperature is large (not shown in the figure), typically comparable with the maximum temperature difference seen at the bottom of Figure 8. Clearly, a firm conclusion on the role of SW electron precipitation on neutral heating must rely on a careful analysis that suppresses other possible sources of temperature variability below $160 \mathrm{~km}$.

\section{Concluding Remarks and Prospects}

SW electron precipitation deposits a substantial amount of energy in the nightside Martian upper atmosphere and causes electron impact ionization in the absence of solar EUV radiation (e.g., Girazian et al., 2017; Lillis et al., 2018; Cui J et al., 2019). It is also likely that such an external energy deposition exerts an influence on the nightside atmospheric thermal structure, a process, however, that has never been explored in previous studies. Here we combine the NGIMS measurements of neutral density (Mahaffy et al., 2015a) and the SWEA measurements of energetic electron intensity (Mitchell et al., 2016), both made on board the recent MAVEN spacecraft, which has allowed us to evaluate, for the first time, the question of SW impact on the nightside thermal structure of the Martian upper atmosphere.

Two different approaches are used to infer upper atmospheric temperatures on the nightside of Mars, providing either mean temperatures from isothermal fittings (e.g., Mahaffy et al., 2015b) or vertical temperature profiles from downward integration of the diffusive equilibrium equation (e.g., Stone et al., 2018; Cui J et al., 2018), both using the NGIMS Ar density data as inputs. The fact that the measured Ar densities are free from wall contamination 


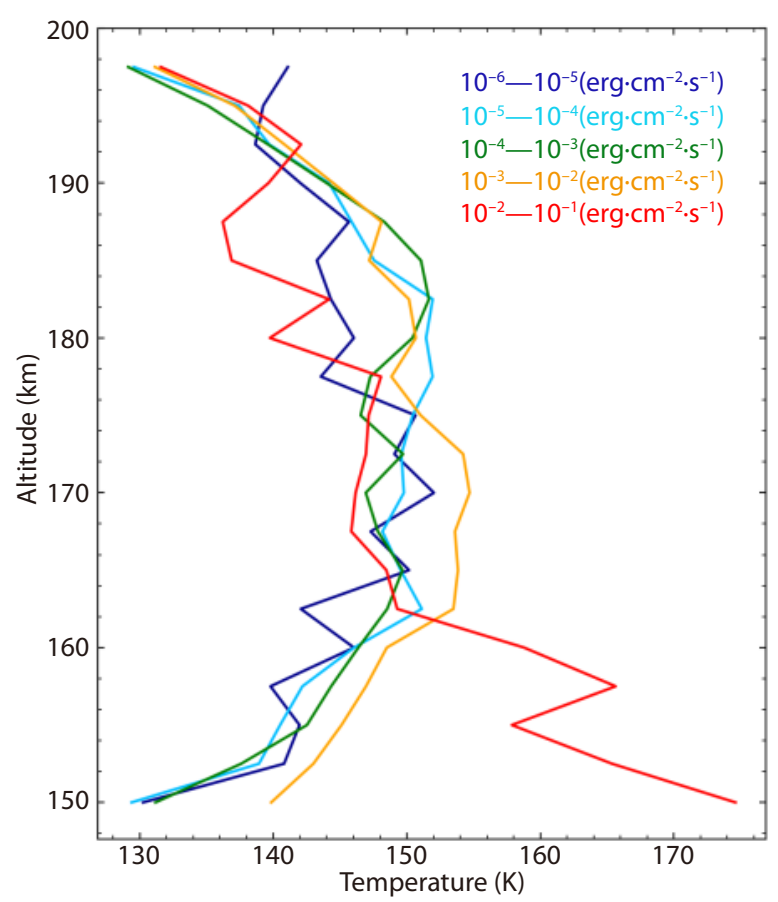

Figure 8. Isothermal neutral temperature in the nightside Martian upper atmosphere as a function of the energetic electron intensity integrated over the altitude range of $150-200 \mathrm{~km}$ and over the energy range of 3-450 eV.

(e.g., Cui J et al., 2009) indicates that both the inbound and outbound measurements could be readily used for the purpose of this study.

The isothermal temperature obtained in the nightside Martian upper atmosphere shows no correlation with either the energetic electron intensity or the crustal magnetic field strength. This suggests that, from a statistical point of view, SW electron precipitation does not exert a noticeable impact on the nightside thermal structure, and further validates the rationale underlying existing global circulation models of the Martian upper atmosphere (e.g. Angelats i Coll et al., 2005; González-Galindo et al., 2005, 2009a, b), of which none have included energy deposition via SW electron precipitation as a viable external energy source. Despite this, our scrutinization of the entire MAVEN data set does reveal individual cases, during $3 \%-4 \%$ of available MAVEN orbits, that are characterized by apparent correlation between patterns of nightside thermal structure and of SW electron precipitation. Such a correlation is commonly manifested as one or several collocated enhancements in both NGIMS-based temperature and SWEA-based electron intensity. Meanwhile, a comparison of mean temperature profiles at different levels of electron intensity indicates that SW-induced neutral heating, if indeed occurring in the nightside Martian upper atmosphere, tends to be effective only below $160 \mathrm{~km}$ and when electron intensities are greater than $0.01 \mathrm{ergs} \cdot \mathrm{cm}^{-2} \cdot \mathrm{s}^{-1}$ integrated over the energy range of 3-450 eV.

Based on the preliminary results presented in this study, we conclude that neutral heating caused by SW electron precipitation can in general be ignored in the nightside Martian upper atmosphere, but should be seriously considered under some extreme circumstances, such as during interplanetary coronal mass ejections (Wilson et al., 2020 and references therein). Part of our analysis in Section 3, leading to the possible identification of SW impact, relies on visual identification of collocated enhancements in both temperature and electron intensity, a procedure that is subject to obvious uncertainties and limitations. A more quantitative and thorough analysis is required to answer more precisely the question of SW effects on the Martian nightside thermal structure. Meanwhile, it is also important that the neutral heating rate due to SW electron precipitation be further parameterized quantitatively with the aid of the combined MAVEN data set, similar to the calculations that we have made for the solar EUV heating rate in the dayside Martian upper atmosphere (Gu H et al., 2020b). Such an extended analysis will be covered in a follow-up investigation.

\section{Acknowledgments}

This work is supported by the B-type Strategic Priority Program No. XDB41000000 funded by the Chinese Academy of Sciences and the pre-research project on Civil Aerospace Technologies No. D020105 funded by China's National Space Administration. The authors also acknowledge support from the National Natural Science Foundation of China through grants 41525015, 41774186, 41904154 , and 42030201. The data used in this work are publicly available at the MAVEN Science Data Center (http://lasp. colorado.edu/maven/sdc/public/).

\section{References}

Acuña, M. H., Connerney, J. E. P., Wasilewski, P., Lin, R. P., Anderson, K. A., Carlson, C. W., McFadden, J., Curtis, D. W., Mitchell, D., ... Ness, N. F. (1998). Magnetic field and plasma observations at mars: Initial results of the mars global surveyor mission. Science, 279(5357), 1676-1680. https://doi.org/10.1126/science.279.5357.1676

Angelats i Coll, M., Forget, F., López-Valverde, M. A., and González-Galindo, F. (2005). The first Mars thermospheric general circulation model: the Martian atmosphere from the ground to 240 km. Geophys. Res. Lett., 32(4), L04201. https://doi.org/10.1029/2004GL021368

Bertaux, J. L., Leblanc, F., Witasse, O., Quemerais, E., Lilensten, J., Stern, S. A., Sandel, B., and Korablev, O. (2005). Discovery of an aurora on Mars. Nature, 435(7043), 790-794. https://doi.org/10.1038/nature03603

Bougher, S. W., and Roble, R. G. (1991). Comparative terrestrial planet thermospheres: 1. Solar cycle variation of global mean temperatures. J. Geophys. Res.: Space Phys., 96(A7), 11045-11055. https://doi.org/10.1029/91JA01162

Bougher, S. W., Engel, S., Roble, R. G., and Foster, B. (1999). Comparative terrestrial planet thermospheres: 2 . Solar cycle variation of global structure and winds at equinox. J. Geophys. Res.: Planets, 104(E7), 16591-16611. https://doi.org/10.1029/1998JE001019

Bougher, S. W., Engel, S., Roble, R. G., and Foster, B. (2000). Comparative terrestrial planet thermospheres: 3 . Solar cycle variation of global structure and winds at solstices. J. Geophys. Res.: Planets, 105(E7), 17669-17692. https://doi.org/10.1029/1999JE001232

Bougher, S. W., Roeten, K. J., Olsen, K., Mahaffy, P. R., Benna, M., Elrod, M., Jain, S. K., Schneider, N. M., Deighan, J., ... Jakosky, B. M. (2017). The structure and variability of Mars dayside thermosphere from MAVEN NGIMS and IUVS measurements: seasonal and solar activity trends in scale heights and temperatures. J. Geophys. Res.: Space Phys., 122(1), 1296-1313. https://doi.org/10.1002/2016JA023454

Bovino, S., Zhang, P., Gianturco, F. A., Dalgarno, A., and Kharchenko, V. (2011). Energy transfer in $\mathrm{O}$ collisions with $\mathrm{He}$ isotopes and helium escape from Mars. Geophys. Res. Lett., 38(2), L02203. https://doi.org/10.1029/2010GL045763 
Connerney, J. E. P., Acunã, M. H., Wasilewski, P. J., Ness, N. F., Rème, H., Mazelle, C., Vignes, D., Lin, R. P., Mitchell, D. L., and Cloutier, P. A. (1999). Magnetic lineations in the ancient crust of mars. Science, 284(5415), 794-798. https://doi.org/10.1126/science.284.5415.794

Cui, J., Yelle, R. V., Vuitton, V., Waite, J. H. Jr., Kasprzak, W. T., Gell, D. A., Niemann, H. B., Müller-Wodarg, I. C. F., Borggren, N., ... Magee, B. A. (2009). Analysis of Titan's neutral upper atmosphere from Cassini Ion Neutral Mass Spectrometer measurements. Icarus, 200(2), 581-615. https://doi.org/10.1016/j.icarus.2008.12.005

Cui, J., Galand, M., Yelle, R. V., Wei, Y., and Zhang, S. J. (2015). Day-to-night transport in the Martian ionosphere: Implications from total electron content measurements. J. Geophys. Res.: Space Phys., 120(3), 2333-2346. https://doi.org/10.1002/2014JA020788

Cui, J., Yelle, R. V., Zhao, L. L., Stone, S., Jiang, F. Y., Cao, Y. T., Yao, M. J., Koskinen, T. T., and Wei, Y. (2018). The impact of crustal magnetic fields on the thermal structure of the Martian upper atmosphere. Astrophys. J. Lett., 853(2), L33. https://doi.org/10.3847/2041-8213/aaa89a

Cui, J., Cao, Y. T., Wu, X. S., Xu, S. S., Yelle, R. V., Stone, S., Vigren, E., Edberg, N. J. T., Shen, C. L., ... Wei, Y. (2019). Evaluating local ionization balance in the nightside Martian upper atmosphere during MAVEN Deep Dip campaigns. Astrophys. J. Lett., 876(1), L12. https://doi.org/10.3847/2041-8213/ab1b34

England, S. L., Liu, G., Yîgit, E., Mahaffy, P. R., Elrod, M., Benna, M., Nakagawa, H., Terada, N., and Jakosky, B. (2017). MAVEN NGIMS observations of atmospheric gravity waves in the Martian thermosphere. J. Geophys. Res.: Space Phys., 122(2), 2310-2335. https://doi.org/10.1002/2016JA023475

Forbes, J. M., Lemoine, F. G., Bruinsma, S. L., Smith, M. D., and Zhang, X. L. (2008). Solar flux variability of Mars' exosphere densities and temperatures. Geophys. Res. Lett., 35(1), L01201. https://doi.org/10.1029/2007GL031904

Forget, F., Montmessin, F., Bertaux, J. L., González-Galindo, F., Lebonnois, S., Quémerais, E., Reberac, A., Dimarellis, E., and López-Valverde, M. A. (2009). Density and temperatures of the upper Martian atmosphere measured by stellar occultations with Mars Express SPICAM. J. Geophys. Res.: Planets, 114(E1), E01004. https://doi.org/10.1029/2008JE003086

Fox, J. L., Johnson, A. S., Ard, S. G., Shuman, N. S., and Viggiano, A. A. (2017). Photochemical determination of $O$ densities in the Martian thermosphere: effect of a revised rate coefficient. Geophys. Res. Lett., 44(16), 8099-8106. https://doi.org/10.1002/2017GL074562

Fu, M. H., Cui, J., Wu, X. S., Wu, Z. P., and Li, J. (2020). The variations of the Martian exobase altitude. Earth Planet. Phys., 4(1), 4-10. https://doi.org/10.26464/epp2020010

Girazian, Z., Mahaffy, P., Lillis, R. J., Benna, M., Elrod, M., Fowler, C. M., and Mitchell, D. L. (2017). Ion densities in the nightside ionosphere of Mars: Effects of electron impact ionization. Geophys. Res. Lett., 44(22), 11248-11256. https://doi.org/10.1002/2017GL075431

González-Galindo, F., López-Valverde, M. A., Angelats i Coll, M., and Forget, F. (2005). Extension of a Martian general circulation model to thermospheric altitudes: UV heating and photochemical models. J. Geophys. Res.: Planets, 110(E9), E09008. https://doi.org/10.1029/2004JE002312

González-Galindo, F., Forget, F., López-Valverde, M. A., Angelats i Coll, M., and Millour, E. (2009a). A ground-to-exosphere Martian general circulation model: 1. Seasonal, diurnal, and solar cycle variation of thermospheric temperatures. J. Geophys. Res.: Planets, 114(E4), E04001. https://doi.org/10.1029/2008JE003246

González-Galindo, F., Forget, F., López-Valverde, M. A., and Angelats i Coll, M. (2009b). A ground-to-exosphere Martian general circulation model: 2 . Atmosphere during solstice conditions-Thermospheric polar warming. J. Geophys. Res.: Planets, 114(E8), E08004. https://doi.org/10.1029/2008JE003277

Gu, H., Cui, J., Niu, D. D., Cao, Y. T., Wu, X. S., Li, J., Wu, Z. P., He, F., and Wei, Y. (2020a). Neutral heating efficiency in the dayside Martian upper atmosphere. Astronom. J., 159(2), 39. https://doi.org/10.3847/1538$3881 / \mathrm{ab} 5 \mathrm{fcc}$

Gu, H., Cui, J., Niu, D. D., He, Z. G., and Li, K. (2020b). Monte Carlo calculations of helium escape on Mars via energy transfer from hot oxygen atoms. Astrophys. J., 902(2), 121. https://doi.org/10.3847/1538-4357/abb6e9 Jain, S. K., Stewart, A. I. F., Schneider, N. M., Deighan, J., Stiepen, A., Evans, J. S.,
Stevens, M. H., Chaffin, M. S., Crismani, M., ... Jakosky, B. M. (2015). The structure and variability of Mars upper atmosphere as seen in MAVEN/IUVS dayglow observations. Geophys. Res. Lett., 42(21), 9023-9030. https://doi.org/10.1002/2015GL065419

Jain, S. K., Deighan, J., Schneider, N. M., Stewart, A. I. F., Evans, J. S., Thiemann, E. M. B., Chaffin, M. S., Crismani, M., Stevens, M. H., ... Jakosky, B. M. (2018). Martian thermospheric response to an X8.2 solar flare on 10 September 2017 as seen by MAVEN/IUVS. Geophys. Res. Lett., 45(15), 7312-7319. https://doi.org/10.1029/2018GL077731

Jakosky, B. M., Grebowsky, J. M., Luhmann, J. G., and Brain, D. A. (2015). Initial results from the MAVEN mission to Mars. Geophys. Res. Lett., 42(21), 8791-8802. https://doi.org/10.1002/2015GL065271

Krasnopolsky, V. A. (2010). Solar activity variations of thermospheric temperatures on Mars and a problem of $\mathrm{CO}$ in the lower atmosphere. Icarus, 207(2), 638-647. https://doi.org/10.1016/j.icarus.2009.12.036

Leclercq, L., Williamson, H. N., Johnson, R. E., Tucker, O. J., Tian, L., and Snowden, D. (2020). Molecular kinetic simulations of transient perturbations in a planet's upper atmosphere. Icarus, 335, 113394. https://doi.org/10.1016/j.icarus.2019.113394

Lillis, R. J., Mitchell, D. L., Steckiewicz, M., Brain, D., Xu, S. S., Weber, T., Halekas, J., Connerney, J., Espley, J., ... Eparvier, F. (2018). Ionizing electrons on the Martian nightside: structure and variability. J. Geophys. Res.: Space Phys., 123(5), 4349-4363. https://doi.org/10.1029/2017JA025151

Magalhães, J. A., Schofield, J. T., and Seiff, A. (1999). Results of the Mars Pathfinder atmospheric structure investigation. J. Geophys. Res.: Planets, 104(E4), 8943-8956. https://doi.org/10.1029/1998JE900041

Mahaffy, P. R., Benna, M., King, T., Harpold, D. N., Arvey, R., Barciniak, M., Bendt, M., Carrigan, D., Errigo, T., ... Nolan, J. T. (2015a). The neutral gas and ion mass spectrometer on the mars atmosphere and volatile evolution mission. Space Sci. Rev., 195(1-4), 49-73. https://doi.org/10.1007/s11214-014-0091-1

Mahaffy, P. R., Benna, M., Elrod, M., Yelle, R. V., Bougher, S. W., Stone, S. W., and Jakosky, B. M. (2015b). Structure and composition of the neutral upper atmosphere of Mars from the MAVEN NGIMS investigation. Geophys. Res. Lett., 42(21), 8951-8957. https://doi.org/10.1002/2015GL065329

Mitchell, D. L., Mazelle, C., Sauvaud, J. A., Thocaven, J. J., Rouzaud, J., Fedorov, A., Rouger, P., Toublanc, D., Taylor, E., ... Jakosky, B. M. (2016). The MAVEN solar wind electron analyzer. Space Sci. Rev., 200(1-4), 495-528. https://doi.org/10.1007/s11214-015-0232-1

Morschhauser, A., Lesur, V., and Grott, M. (2014). A spherical harmonic model of the lithospheric magnetic field of Mars. J. Geophys. Res.: Planets, 119(6), 1162-1188. https://doi.org/10.1002/2013JE004555

Niu, D. D., Cui, J., Gu, H., Wu, X. S., Wu, S. Q., Lu, H. Y., Chai, L. H., and Wei, Y. (2020). Energetic Electron depletions in the nightside Martian upper atmosphere revisited. J. Geophys. Res.: Space Phys., 125(4), e2019JA027670. https://doi.org/10.1029/2019JA027670

Pilinski, M., Bougher, S., Greer, K., Thiemann, E., Andersson, L., Benna, M., and Elrod, M. (2018). First Evidence of persistent nighttime temperature structures in the neutral thermosphere of Mars. Geophys. Res. Lett., 45(17), 8819-8825. https://doi.org/10.1029/2018GL078761

Schofield, J. T., Barnes, J. R., Crisp, D., Haberle, R. M., Larsen, S., Magalhães, J. A., Murphy, J. R., Seiff, A., and Wilson, G. (1997). The mars pathfinder atmospheric structure investigation/meteorology (ASI/MET) experiment. Science, 278(5344), 1752-1758. https://doi.org/10.1126/science.278.5344.1752

Siddle, A. G., Mueller-Wodarg, I. C. F., Stone, S. W., and Yelle, R. V. (2019). Global characteristics of gravity waves in the upper atmosphere of Mars as measured by MAVEN/NGIMS. Icarus, 333, 12-21. https://doi.org/10.1016/j.icarus.2019.05.021

Slipski, M., Jakosky, B. M., Benna, M., Elrod, M., Mahaffy, P., Kass, D., Stone, S., and Yelle, R. (2018). Variability of Martian turbopause altitudes. J. Geophys. Res.: Planet, 123(11), 2939-2957. https://doi.org/10.1029/2018JE005704

Steckiewicz, M., Mazelle, C., Garnier, P., André, N., Penou, E., Beth, A., Sauvaud, J. A., Toublanc, D., Mitchell, D. L., ... Jakosky, B. M. (2015). Altitude dependence of nightside Martian suprathermal electron depletions as revealed by MAVEN observations. Geophys. Res. Lett., 42(21), 8877-8884. https://doi.org/10.1002/2015GL065257 
Steckiewicz, M., Garnier, P., André, N., Mitchell, D. L., Andersson, L., Penou, E., Beth, A., Fedorov, A., Sauvaud, J. A., ... Jakosky, B. M. (2017). Comparative study of the Martian suprathermal electron depletions based on Mars Global Surveyor, Mars Express, and Mars Atmosphere and Volatile EvolutioN mission observations. J. Geophys. Res.: Space Phys., 122(1), 857-873. https://doi.org/10.1002/2016JA023205

Stiepen, A., Gérard, J. C., Bougher, S., Montmessin, F., Hubert, B., and Bertaux, J. L. (2015). Mars thermospheric scale height: $\mathrm{CO}$ Cameron and $\mathrm{CO}_{2}{ }^{+}$dayglow observations from Mars Express. Icarus, 245, 295-305. https://doi.org/10.1016/j.icarus.2014.09.051

Stone, S. W., Yelle, R. V., Benna, M., Elrod, M. K., and Mahaffy, P. R. (2018). Thermal structure of the Martian upper atmosphere from MAVEN NGIMS. J. Geophys. Res.: Planets, 123(11), 2842-2867. https://doi.org/10.1029/2018JE005559

Wilson, L. B., Chen, L. J., Wang, S., Schwartz, S. J., Turner, D. L., Stevens, M. L.,
Kasper, J. C., Osmane, A., Caprioli, D., ... and Goodrich, K. A. (2020). Electron energy partition across interplanetary shocks. III. Analysis. Astrophys. J., 893(1), 22. https://doi.org/10.3847/1538-4357/ab7d39

Wu, X. S., Cui, J., Yelle, R. V., Cao, Y. T., He, Z. G., He, F., and Wei, Y. (2020). Photoelectrons as a tracer of planetary atmospheric composition: application to CO on Mars. J. Geophys. Res.: Planets, 125(7), e2020JE006441. https://doi.org/10.1029/2020JE006441

Zhang, Q., Gu, H., Cui, J., Cheng, Y. M., He, Z. G., Zhong, J. H., He, F., and Wei, Y. (2020). Atomic oxygen escape on Mars driven by electron impact excitation and ionization. Astronom. J., 159(2), 54. https://doi.org/10.3847/15383881/ab6297

Zurek, R. W., Tolson, R. A., Bougher, S. W., Lugo, R. A., Baird, D. T., Bell, J. M., and Jakosky, B. M. (2017). Mars thermosphere as seen in MAVEN accelerometer data. J. Geophys. Res.: Space Phys., 122(3), 3798-3814. https://doi.org/10.1002/2016JA023641 